\title{
Conhecimento de enfermeiros acerca da terapia nutricional
}

\section{Knowledge of nurses about nutritional therapy}

\section{Jaqueline Almeida Guimarães Barbosa' ${ }^{1}$ (1) Clara Marize Carlos ${ }^{2}$ (1) Raquel Fernandes Costa ${ }^{3}$ (1) Giovana Paula Rezende Simino 4 (1)}

${ }^{1}$ Autora para correspondência. Universidade Federal de Minas Gerais (Belo Horizonte). Minas Gerais, Brasil. jaqueline@task.com.br ${ }^{2-4}$ Universidade Federal de Minas Gerais (Belo Horizonte). Minas Gerais, Brasil. claramarize@gmail.com, raquel.costa@hc.ufmg.br, gsimino@yahoo.com.br

RESUMO | OBJETIVO: Avaliar o conhecimento de enfermeiros de um hospital geral de ensino e alta complexidade sobre a terapia nutricional e os cuidados aos pacientes em uso da mesma. MÉTODO: Estudo quantitativo, observacional, descritivo, realizado com 39 enfermeiros. Foi aplicado questionário e realizadas análises estatísticas descritivas. RESULTADOS: O conhecimento de aspectos considerados essenciais sobre terapia nutricional enteral e parenteral alcançou taxas de acerto superior a $60 \%$ na maioria das questões levantadas. Contudo, identificou-se haver divergências acerca da realização de procedimentos relativos a essa terapêutica, como de inserção do cateter nasogástrico e avaliação da estase gástrica. A compreensão acerca das gastro e jejunostomias mostrou-se limitada, o que pode comprometer os cuidados aos pacientes. CONCLUSÃO: O conhecimento dos enfermeiros acerca de aspectos básicos da terapia nutricional pode ser considerado, de forma geral, satisfatório, devendo ser continuadamente atualizado e aprimorado visando contribuir para uma prática refletida, segura e padronizada.

DESCRITORES: Terapia nutricional. Cuidados de enfermagem. Conhecimento. Nutrição enteral. Nutrição parenteral.

\begin{abstract}
OBJECTIVE: To evaluate the knowledge of nurses of a general teaching hospital and high complexity on nutritional therapy and care for patients using it. METHOD: Quantitative, descriptive, observational study conducted with 39 nurses. A questionnaire was applied and descriptive statistical analyses were performed. RESULTS: The knowledge of aspects considered essential on enteral and parenteral nutritional therapy reached rates of accuracy higher than $60 \%$ for most questions raised. However, there were differences regarding the performance of procedures related to this therapy, such as insertion of the nasogastric catheter and evaluation of gastric stasis. The understanding of the gastro and jejunostomy showed to be limited, which can compromise patient care. CONCLUSION: The knowledge of nurses about basic aspects of nutritional therapy can be considered, in general, satisfactory, and should be continuously updated and improved in order to contribute to a safe, reflected and standardized practice.
\end{abstract}

DESCRIPTORS: Nutritional therapy. Nursing care. Knowledge. Enteral nutrition. Parenteral nutrition. 


\section{Introdução}

A alimentação é essencial não só ao crescimento, mas à manutenção do funcionamento do organismo ao longo da vida. O ser humano adulto,quando saudável,tem habilidades para assegurar sua alimentação de forma autônoma. Contudo, em alguns momentos, essa capacidade pode estar afetada, seja pela presença de doenças ou sintomas a elas associadas, como náuseas e vômitos, bem como decorrente de tratamentos que muitas vezes impossibilitam a ingestão de alimentos, como em situações de uso de ventilação mecânica em unidade de terapia intensiva.

Nos hospitais gerais e principalmente naqueles que realizam atendimento de média e alta complexidade, não é raro deparar-se com pacientes que já apresentam acometimento do seu estado nutricional no ato da internação. O mais importante estudo brasileiro já realizado sobre o assunto no Brasil, no qual foram avaliados4000 pacientes em diferentes estados, encontrou que $48,1 \%$ dos pacientes hospitalizados encontravam-se desnutridos, e que esse quadro agravava-se com o aumento do tempo de internação. A desnutrição chegou a atingir $60 \%$ dos pacientes hospitalizados a mais de 15 dias¹.

Estudos comprovam que pacientes desnutridos tem afetados a sua resposta imunológica, ficando mais vulneráveis para desenvolver infecções e lesões por pressão. Além disso, apresentam maior dificuldade para cicatrização, redução da mobilidade dos músculos respiratórios e fadiga, dentre outros, sendo que um comprometimento ocasiona outros sucessivamen$t^{2}$. Para enfrentar essa problemática dispõe-se da Terapia Nutricional (TN), definida como um conjunto de procedimentos terapêuticos para manutenção ou recuperação do estado nutricional do paciente.

A TN inclui a suplementação oral, a Terapia Nutricional Enteral (TNE) e a Terapia Nutricional Parenteral (TNP), ou a combinação de dessas terapêuticas simultaneamente, sendo que sua regulamentação no Brasil exige que seja realizada por uma equipe multiprofisisonal $^{3,4}$. É uma terapêutica efetiva, mas não isenta de riscos, que podem ser graves e até fatais. Apesar da literatura sobre da ocorrência de eventos adversos relacionados ao uso da TN ser incipiente, relatórios governamentais mostram falhas na administração de dietas como a sexta causa de eventos adversos, com 1713 ocorrências 5 .
Faz-se necessário, pois, que toda a equipe de saúde e em especial a equipe de enfermagem compreenda o uso dessa terapêutica, não só quanto aos cuidados com o paciente, mas englobando sua finalidade, indicações e os riscos envolvidos, a fim de que suas práticas assistenciais sejam executadas de forma refletida e efetiva. Estudos mostram que os profissionais de saúde, incluindo médicos, enfermeiros e técnicos de enfermagem, apresentam conhecimentos insuficientes acerca da TN, o que pode favorecer a ocorrência de complicações e o alcance dos resultados esperados ${ }^{6-8}$. Identificou-se uma lacuna quanto a estudos dessa natureza realizados especificamente com enfermeiros no Brasil, considerando o papel que Ihe cabe na terapia nutricional, o que desencadeou a realização desta pesquisa.

Sua realização justifica-se, inclusive, por ser realizado em um hospital de ensino, lócus de difusão do saber-fazer, no qual as práticas assistenciais devem ser qualificadas, uma vez que são observadas por discentes em fase de formação, podendo ser seguidas ao longo de toda a vida profissional. Assim, esse estudo teve como objetivo identificar o conhecimento de enfermeiros de um hospital de ensino e alta complexidade sobre a terapia nutricional e os cuidados aos pacientes em uso da mesma. Os resultados poderão contribuir com informações que possam subsidiar o aprimoramento da prática assistencial em terapia nutricional, incluindo a formação do enfermeiro.

\section{Método}

Estudo quantitativo, observacional, descritivo. Os participantes foram enfermeiros que trabalham em um hospital geral, público, de ensino, de grande porte e alta complexidade. Foram incluídos no estudo enfermeiros que atuantes em qualquer unidade do hospital, tendo em vista que os profissionais podem mudar de setor conforme necessário. Como os enfermeiros trabalham por regime de escala de plantão, o universo do estudo foi contemplado por profissionais que atuavam no turno diurno, e que se interessasse em participar da pesquisa, em amostragem por conveniência. 
O hospital onde se deu a coleta de dados tem em torno de 480 leitos, sendo em torno de 50 leitos destinados a tratamento intensivo. É referência em tratamento oncológico, cirurgia cardíaca e transplantes, além de maternidade e berçário de alto risco. Realiza em torno de 1600 cirurgias mensais. Na instituição há Equipe Multiprofissional de Terapia Nutricional (EMTN) a qual normatiza e monitora o uso dessa terapêutica e realiza capacitações da equipe de enfermagem. Os profissionais ingressam por meio de concurso.

Os participantes do estudo foram convidados a participar da pesquisa após esclarecimentos sobre o objetivo da mesma e assinatura do Termo de Consentimento Livre e Esclarecido (TCLE), conforme exigência da Resolução 466/12 do CNS. A coleta de dados se deu anteriormente à realização de uma capacitação em serviço voltada para enfermeiros, em auditório amplo que assegurava a privacidade dos participantes para responder às questões, tendo sido realizada em 2018. O trabalho foi aprovado pelo COEP da UFMG, parecer n. 2.232.124, CAAE: 72683417.3.0000.5149.

Inicialmente buscou-se na literatura algum instrumento validado sobre o assunto para ser aplicado e que pudesse atender aos objetivos do estudo, não tendo sido encontrado. Assim, um questionário foi elaborado por um enfermeiro especialista em terapia nutricional, o qual foi revisado e avaliado e validado por outro enfermeiro também especialista na área.

O questionário foi composto de questões fechadas, as quais abordavam a indicação e contra-indicação do uso da TN enteral e parenteral bem como das vias de infusão, as principais complicações e riscos envolvidos no uso dessa terapêutica os cuidados de enfermagem necessários para sua prevenção. Em duas questões havia um campo aberto para que apresentassem a justificativa para a resposta escolhida, visando maior aprofundamento acerca dos conhecimentos sobre o assunto investigado.
O questionário foi composto também de duas questões abertas, mas de cunho objetivas, inseridas com o propósito de evitar que respostas pré-estabelecidas em questões fechadas pudessem ocultar a 'originalidade' das respostas. Essas questões indagavam procedimentos realizados pelo enfermeiro relacionados ao uso da terapia nutricional, como acerca da medida utilizada para a inserção do cateter nasogástrico e dos parâmetros adotados na avaliação da estase gástrica.

Foram coletados, ainda, dados que permitissem a caracterização do perfil dos participantes, como setor de atuação, pós-graduação, e tempo de atuação profissional. Realizaram-se análises estatísticas descritivas dos dados coletados. Quando as respostas corretas foram respondidas por mais de $80 \%$ dos participantes, o conhecimento no assunto foi considerado muito bom. Entre 60 e $79 \%$ de acerto o conhecimento foi considerado satisfatório. E quando o percentual de os acertos não ultrapassou 59\%,esses foram considerados insuficientes.

\section{Resultados}

Participaram da pesquisa 39 enfermeiros, sendo que a maioria $(46,2 \%)$ tinha entre 5 e 10 anos de formado, $71,8 \%$ eram especialistas dos quais a maior parte era em terapia intensiva (28,2\%). As demais especialidades variaram de maneira homogênea entre transplante de órgãos, estomaterapia, oncologia, nefrologia, educação em saúde, controle de infecção e em auditoria de serviços de saúde. A maioria (46\%) atuava em Unidade de Terapia Intensiva e 38,7\% em unidades de internação. Nenhum participante tinha período de atuação na instituição inferior a um ano, o que leva a crer que todos já conhecessem os protocolos institucionais e já tivessem tido a oportunidade de participar de algum treinamento sobre essa terapêutica (Tabela 1). 
Tabela 1. Características quanto à formação e setor de atuação dos participantes (n=39). Belo Horizonte, MG, Brasil, 2019

\begin{tabular}{lr}
\hline Variáveis & $\mathbf{N}(\%)$ \\
\hline Tempo de formado & $5(12,8)$ \\
$0-5$ anos & $18(46,2)$ \\
$6-10$ & $10(25,7)$ \\
Mais de 11 anos & $6(15,3)$ \\
Não responderam & \\
Formação & $11(28,2)$ \\
Graduação & $28(71,8)$ \\
Pós-graduação & \\
Setor de atuação & $18(46,2)$ \\
UTI & $15(38,7)$ \\
Unidade de internação & $6(15,3)$ \\
Unidade de pronto atendimento & \\
\hline
\end{tabular}

Fonte: Dados do autor

Ao serem indagados acerca dos riscos e complicações envolvidos no uso da TNP, $89 \%$ dos participantes assinalaram como verdadeiro que a TNP é uma terapêutica que oferece alto risco aos pacientes, o que foi justificado em campo aberto pelo fato de ser infundida na corrente sanguínea, e, portanto, com risco de infecção. Outras potenciais complicações no uso da TNP foram citadas, como embolia e alergia, mas por uma minoria (31\%). A necessidade de manipulação asséptica da NP mostrou-se ser do conhecimento de todos os participantes, bem como a necessidade de se ter uma via exclusiva para infusão da NP.

Quando questionados acerca das diferenças existentes entre a solução de nutrição parenteral infundida em acesso periférico daquela infundida em acesso venoso central e suas indicações, a maioria $(97,4 \%)$ demonstrou conhecer que estas soluções apresentam diferenças na osmolaridade. Além disso, esse mesmo percentual tem clareza que a nutrição parenteral administrada em via periférica apresenta menor risco de complicações sistêmicas quando comparado à nutrição parenteral em acesso central.

Quanto aos cuidados aos pacientes em uso de TNP, foi consenso no grupo a percepção de ser necessário monitorar o acesso de infusão da solução quanto à presença de sinais flogísticos e de infiltração, e da necessidade de troca de equipo a cada novo frasco de nutrição parenteral a ser instalado. Contudo, somente $31 \%$ demonstraram conhecimento acerca da necessidade de se observar a solução de nutrição parenteral em infusão quanto ao seu aspecto, atentando para separação de fases.
Em relação ao uso da TNE, foi consenso entre os participantes ser esta a opção mais fisiológica quando a TN for necessária. Quanto às indicações para o uso da terapia nutricional, $74,3 \%$ sinalizaram corretamente a alternativa que incluía pacientes com sequelas de acidente vascular encefálico e mal de Alzheimer avançado, bem como em situações de hipercatabolismo.

Em se tratando do uso de ostomias de alimentação, a maioria $(76,9 \%)$ tem clareza que sua indicação se dá para pacientes com previsão de mais de 4 semanas de uso da TNE. Entre as justificativas para a escolha pela confecção de gastro ou jejunostomias os participantes pontuaram o menor risco de obstrução do cateter e de broncoaspiração quando comparado ao uso do cateter nasogástrico, bem como de problemas com o posicionamento e perda do acesso.

Ao se abordar os fatores associados às complicações possíveis no uso de gastro e jejunostomias, observou-se ser esse um conhecimento restrito a parte dos participantes do estudo. Pouco mais da metade dos participantes $(56,4 \%)$ demonstrou conhecer que a realização de gastrostomia por via endoscópica e o uso de sondas próprias oferece menores riscos de complicações peri-estoma. Além disso, 20\% não consideraram que a desnutrição prévia seja fator favorecedor da ocorrência de complicações nas ostomias de alimentação.

Em questão do tipo aberta acerca da medida adotada para posicionamento do cateter nasogástrico, divergências foram identificadas. Entre os participantes, $28,1 \%$ afirmaram ser 'Ponta do nariz ao lóbulo da 
orelha, e deste ao processo xifoide, com acréscimo de mais 15 a 20 cm;; 20,5\% responderam adotar a medida 'Ponta do nariz ao lóbulo da orelha, e deste ao processo xifoide'; 20,5\% afirmaram ser "Ponta do nariz ao lóbulo da orelha, e deste ao processo xifóide mais $10 \mathrm{~cm}$; e 2,5\% afirmaram acrescentar $5 \mathrm{~cm}$ posterior ao processo xifoide. Chamou a atenção o fato de $28,3 \%$ não ter respondido à questão, o que se pode se dar por receio em apresentar resposta com erro.

No que diz respeito aos cuidados com o paciente em uso de CNE, 97,4\% dos participantes assinalaram a necessidade de manutenção do paciente com a cabeceira elevada e de se reavaliar a fixação do mesmo diariamente. Além disso, 94\% demonstraram compreensão do uso de equipo de cor diferenciada para a administração da nutrição enteral e da necessidade de troca dos mesmos a cada troca de frasco, sendo que na Instituição só é utilizado sistema fechado em pacientes adultos. Esse mesmo percentual assinalou como verdadeiro a importância de não se administrar mais de um medicamento concomitantemente pelo cateter, e da necessidade de irrigação deste com água periodicamente. Contudo, para 31\% a conferência diária do posicionamento do CNE não se faz necessário.

No levantamento feito em questão aberta acerca do volume de estase gástrica que consideram ser indicativo da interrupção da infusão da nutrição enteral, observou-se novamente haver divergências. A maioria $(53,8 \%)$ respondeu ser um volume residual maior que $250 \mathrm{ml} ; 7,6 \%$ afirmou ser acima de $200 \mathrm{ml} ; 7,6 \%$ ser superior a $300 \mathrm{ml} ; 5,1 \%$ ser o volume correspondente a $1 / 3$ do volume de dieta infundido na última hora; $5,1 \%$ ser o volume superior a $100 \mathrm{ml} ; 5,1 \%$ superior a 500 $\mathrm{ml} ; 2,5 \%$ ser acima de $50 \mathrm{ml} ; 2,5 \%$ acima de $150 \mathrm{ml}$; $2,5 \%$ ser $50 \%$ do volume administrado na última hora; $2,5 \%$ ser o volume correspondente ao total infundido na última hora; 5\% não responderam à questão.

\section{Discussão}

Os achados mostram que os participantes possuem, de forma geral, conhecimentos essenciais quanto ao uso da TNE e TNP, mediante o percentual de acerto superior a $60 \%$ para a maioria das questões colocadas, conhecimentos estes que estão dentro do recomendado pela literatura e legislação vigente ${ }^{3-4,9}$. Este resultado pode ser resultante de capacitações realizadas pela EMTN na Instituição, uma vez que $46 \%$ tinham entre 6 e 10 anos de trabalho na Instituição, bem como da estabilidade do quadro de profissionais e do estímulo à realização de pós-graduação na Instituição, cursada por $71 \%$ dos participantes. Além disso, $46 \%$ atuavam em UTI, setor este no qual o uso da TN é mais frequente.

Cabe pontuar que o enfermeiro deve participar do processo de preparação e orientação do paciente para o uso da TN, bem como das escolhas de como deve ser implementada, tendo em vista que é quem mais conhece o paciente e sabe das dificuldades que podem advir para a manutenção da terapêutica não só no hospital, mas para aqueles que continuarão a TN no domicílio. Para isso, o enfermeiro deve possuir conhecimentos ampliados e atualizados quanto à TN como um todo.

Todavia, lacunas no conhecimento foram identificadas principalmente quanto às ostomias de alimentação e acerca de cuidados como da necessidade de se confirmar o posicionamento do cateter nasogástrico periodicamente. Em relação à TNP, somente uma minoria tem percepção da necessidade de se avaliar a solução em infusão e acerca da existência de riscos como embolia e alergia. Além disso, as divergências relativas a procedimentos como a inserção do cateter nasogástrico e avaliação da estase gástrica precisam ser discutidas para que essas terapêuticas sejam realizadas de forma segura, consciente e refletida ${ }^{3-4,9}$.

As divergências encontradas evidenciam o não seguimento do protocolo institucional, e refletem divergências existentes também na literatura. Em se tratando do local para posicionamento da sonda de alimentação no estômago, a literatura recomenda a extremidade distal no antro, o que favorece o processo de digestão dos nutrientes e a possível prevenção de refluxo gastroesofágico ${ }^{9}$. Para isso, a medida indicada em obra clássica na Enfermagem é determinada pela ponta do nariz ao lóbulo da orelha até o processo xifoide, com acréscimo de $15 \mathrm{~cm}$ para posicionamento gástrico e 20 a $25 \mathrm{~cm}$ para posicionamento intestinal, o qual pontua,contudo, não haver estudos que confirmem esse técnica como confiável ${ }^{9}$.

A medida indicada para o posicionamento do cateter nasogástrico é 'ponta do nariz ao lóbulo da orelha até o processo xifóide, mais $10 \mathrm{~cm}$ para se ter posicionamento gástrico e 20 a $30 \mathrm{~cm}$ para enteral ${ }^{10}$. Cabe pontuar que o posicionamento do cateter para administração da nutrição enteral em posição pós-pilórica tem 
indicação somente para pacientes com maior risco de aspiração, devendo ser inserido por via endoscópica para que seu posicionamento seja assegurado?.

Em recente ensaio clínico randomizado realizado com 240 participantes, onde foram avaliadas três medidas de posicionamento do cateter nasogástrico para alimentação, encontrou-se que à medida que vai do lóbulo da orelha ao apêndice xifoide e deste ao ponto médio da cicatriz umbilical foi considerada como a que melhor alcançou o posicionamento adequado, no corpo gástrico. A medida 'ponta do nariz ao lóbulo da orelha até o apêndice xifoide, e deste ao ponto médio da cicatriz umbilical' também foi considerada satisfatória, sendo ambas consideradas como de menor risco de aspiração quando comparados com a medida 'ponta do nariz ao lóbulo da orelha e desta ao apêndice xifoide' ${ }^{\prime 11}$.

As controvérsias demonstram a complexidade do procedimento que é da responsabilidade dos enfermeiros, salientando a necessidade de novos estudos sobre a temática a fim de se evitar danos decorrentes de posicionamento incorreto do cateter. Quando a medida ultrapassa o necessário, pode levar uma laçada do cateter dentro do estômago, gerando complicações para a retirada do mesmo, ou também seu retorno ao esôfago, como em um movimento em forma de "u". Já a inserção aquém do necessário pode ocasionar risco de aspiração ${ }^{9,12}$.

Em estudo realizado com diferentes profissionais da área da saúde de uma UTI, dentre os quais enfermeiros e técnicos de enfermagem (58\% dos participantes), $62 \%$ afirmaram sentirem-se preparados tecnicamente para atuar na área de nutrição enteral e parenteral, mas 94\% afirmaram ser necessário e importante receber mais capacitação nessa área ${ }^{13}$.

Em outro estudo, realizado em UTIs na Índia, encontrou-se que os enfermeiros consideram ter consciência sobre a importância da TNE e conhecimentos sobre os cuidados aos pacientes em uso da mesma. Nesse estudo, o volume considerado na avaliação da estase gástrica variou entre os profissionais, corroborando com os achados desse estudo ${ }^{14}$.

O volume residual gástrico considerado como impeditivo da continuidade da infusão da nutrição enteral não tem consenso na literatura. O retardo no esvaziamento gástrico favorece o refluxo e a aspiração, o que requer que o paciente seja avaliado para se evitar essas complicações. Além disso, o conteúdo deve ser observado quanto ao volume e aspecto, sendo um dado importante para as intervenções da EMTN ${ }^{9,10}$.

Pacientes críticos têm maior probabilidade da ocorrência de estase gástrica, decorrente muitas vezes da própria instabilidade clínica que leva esses pacientes a precisarem do tratamento intensivo. Cabe pontuar, contudo, que o volume residual gástrico deve ser avaliado juntamente a outros dados clínicos do paciente, como a distensão abdominal e ruídos hidroaéreos. Além disso, desprezar o resíduo gástrico pode propiciar ou agravar o desequilíbrio hidroeletrolítico e as alterações do balanço nutricional, sinalizando a importância da atuação multiprofissional e interdisciplinar na $\mathrm{TN}^{9,10}$.

A TN é uma terapêutica complexa e por isso deveria merecer a priorização da atenção do enfermeiro aos pacientes em uso das mesmas, o que nem sempre ocorre e reflete o preparo insuficiente dos enfermeiros no assunto. Em estudo realizado com 359 respondentes atuantes em UTI na Austrália, identificou-se que os enfermeiros consideravam a TN importante, mas possuíam significativo déficit de conhecimento relativo à mesma, o que se inferiu ser uma das causas da baixa priorização conferida a essa modalidade terapêutica entre os cuidados prestados aos pacientes. No estudo, concluiu-se que essa situação refletia em atrasos na infusão da TN, contribuindo para o agravamento da desnutrição ${ }^{15}$.

A indústria farmacêutica tem disponibilizado muitas inovações na área de TN visando evitar erros e danos aos pacientes. Contudo, essas inovações não isentam necessidade de capacitação dos enfermeiros, a partir das evidências científicas mais recentes, e do monitoramento quanto à qualidade da assistência prestada ${ }^{16-17}$. As divergências encontradas nas questões abertas demonstram a importância da realização desse tipo de levantamento, o qual evidencia com maior exatidão os conhecimentos de determinadas temáticas.

Não foram encontrados estudos com questões similares que pudessem servir de comparação com os achados dessa pesquisa, o que acabou por dificultar a discussão dos resultados. As limitações do estudo estão em não ter sido usado instrumento validado, por não ter sido encontrado, e pela amostragem por conveniência. Entretanto, os resultados poderão auxiliar enfermeiros de outras instituições a refletirem 
acerca de aspectos importantes a serem abordados nas capacitações em serviço.

\section{Conclusão}

O conhecimento dos enfermeiros acerca de aspectos essenciais da terapia nutricional e quanto aos cuidados de enfermagem aos pacientes em uso de terapia nutricional enteral e parenteral mostrou-se em sua maioria satisfatório. Contudo, alguns aspectos precisam ser aprimorados a fim de assegurar uma assistência em TN efetiva e isenta de danos, dentre os quais se ressalta a compreensão mais ampliada acerca das ostomias de alimentação, uma vez podem contribuir na tomada de decisões junto à equipe multiprofissional em prol de evitar complicações futuras aos pacientes.

Soma-se a isso a ser necessário capacitar permanentemente os profissionais para o seguimento dos protocolos institucionais, os quais devem ser atualizados com base nas evidências mais recentes. Acredita-se que os resultados encontrados nesse estudo possam contribuir inclusive para o ensino na graduação em enfermagem, uma vez que as fragilidades identificadas no conhecimento dos profissionais refletem faIhas na formação profissional.

\section{Contribuições dos autores}

Barbosa JAG foi responsável pelo delineamento, análise dos resultados e redação do artigo. Carlos CM e Costa RF foram responsáveis pela concepção, coleta de dados e redação final. Simino GPR foi responsável pela interpretação dos dados e redação final.

\section{Conflitos de interesses}

Nenhum conflito financeiro, legal ou político envolvendo terceiros (governo, empresas e fundações privadas, etc.) foi declarado para nenhum aspecto do trabalho submetido (incluindo, mas não se limitando a subvenções e financiamentos, participação em conselho consultivo, desenho de estudo, preparação de manuscrito, análise estatística, etc.).

\section{Referências}

1. Waitzberg DL, Caiaffa WT, Correia MI. Hospital malnutrition: the Brazilian national survey (IBRANUTRI): a study of 4000 patients. Nutrition. 2001;17(7-8):573-80. doi: 10.1016/s0899-9007(01)00573-1

2. Barker LA, Gout BS, Crowe TC. Hospital malnutrition: prevalence, identification and impact on patients and the healthcare system. Int J Environ res Public Health. 2011;8(2):51427. doi: $10.3390 /$ ijerph8020514

3. Brasil. Ministério da Saúde. Agência Nacional de Vigilância Sanitária. Resolução da diretoria Colegiada, $n^{\circ} 63$ de 6 de julho de 2000. Regulamento Técnico para a Terapia de Nutrição Enteral. Diário oficial- República Federativa do Brasil, Brasília, DF, 6 de julho de 2000.

4. Brasil. Ministério da Saúde. Secretaria de Vigilância Sanitária. Portaria $n^{\circ} 272$, de 8 de abril de 1998. Dispõe sobre o regulamento técnico para a Terapia de Nutrição parenteral. 1998.

5. Brasil. Ministério da saúde. Relatórios dos Estados- Eventos Adversos. [Internet]. 2017. Disponível em: https://www20.anvisa. gov.br/segurancadopaciente/index.php/publicacoes/category/ relatorios-dos-estados

6. Santos AFL, Carvalho AC, Silva IS, Oliveira AS. Conhecimento do enfermeiro sobre o cuidado na administração da nutrição enteral e parenteral. Rev Interd. 2013;6(4);44-50.

7. Porto PA, Mendonça SS. Conhecimento dos profissionais de enfermagem e médicos de um hospital público sobre terapia nutricional. Rev Bras Nutr Clin 2015;30(3):227-34.

8. Paulo DA, Oliveira BMR, Wang DWM, Guimarães MP, Cukier C, Lopes Filho GJ. Conhecimento e atitudes de cirurgiões frente aos conceitos de terapia nutricional. Rev Col Bras Cir. 2013;40(5):409419. doi: $10.1590 /$ S0100-69912013000500011

9. Hinkle JL, Cheever KH. Brunner \& Suddart: Tratado de Enfermagem Médico-Cirúrgica. 13.ed. Rio de Janeiro: Guanabara Koogan; 2016.

10. Oliveira RG. Blackbook da Enfermagem. Belo Horizonte: BlackBook editor; 2016.

11. Santos SCVO. Validação do método preditivo para introdução da sonda nasogástrica na alimentação em adultos: ensaio clínico randomizado [tese]. Campinas: Universidade Estadual de Campinas; 2016.

12. Malta MA, Carvalho-Júnior AF, Andreollo NA, Freitas MIP. Medidas Antropométricas na introdução da sonda nasogástrica para nutrição enteral empregando a Esofagogastroduodenoscopia. ABCD Arq Bras Cir Dig. 2013;26(2):107-111. doi: 10.1590/S0102-67202013000200007 
13. Silva RF, Novaes MRGC, Magalhães DMS. Conhecimento dos profissionais de saúde sobre procedimentos e interações medicamentosas em terapia nutricional. Com Ciências Saúde. 2014;24(3):231-238.

14. Gupta B, Agrawal P, Soni KD, Yadav V, Dhakal R, Khurana $S$ et al. Enteral nutrition practices in the intensive care unit: Understanding of nursing practices and perspectives. J Anaesthesiol Clin Pharmacol. 2012;28(1):41-4. doi: 10.4103/09709185.92433

15. Bloomer MJ, Clarke AB, Morph J. Prioritization of enteral nutrition in intensive care units: a national survey. Nurs Crit Care. 2018;23(3):152-158. doi: 10.1111/nicc.12284

16. Matsuba CST, Ciosak SI. Movimento pela segurança na terapia nutricional enteral: o que há de novo com os dispositivos? BRASPEN J. 2017;32(2):175-82.

17. Kim H, Chang SJ. Implementing an educational program to improve critical care nurses' enteral nutritional support. Aust Crit Care. 2019;32(3):218-222. doi: 10.1016/j.aucc.2018.04.001 